\title{
Towards an ontology modeling tool. A validation in software engineering scenarios
}

\author{
Francisco José García-Peñalvo ${ }^{\mathrm{a}}$, Ricardo Colomo-Palacios ${ }^{\mathrm{b}, *}$, Juan García ${ }^{\mathrm{a}}$, Roberto Therón ${ }^{\mathrm{a}}$ \\ ${ }^{a}$ Universidad de Salamanca, Spain \\ ${ }^{\mathrm{b}}$ Universidad Carlos III de Madrid, Spain
}

\section{A R T I C L E I N F O}

Keywords:

OWL

Ontologies

Knowledge engineering

Visual analytics

Software engineering

\begin{abstract}
A B S T R A C T
Ontology creation and management related processes are very important to define and develop semantic services. Ontology Engineering is the research field that provides the mechanisms to manage the life cycle of the ontologies. However, the process of building ontologies can be tedious and sometimes exhaustive. OWL VisMod is a tool designed for developing ontological engineering based on visual analytics concep tual modeling for OWL ontologies life cycle management, supporting both creation and understanding tasks. This paper is devoted to evaluate OWL VisMod through a set of defined tasks. The same tasks also will be done with the most known tool in Ontology Engineering, Protégé, in order to compare the obtained results and be able to know how is OWL VisMod perceived for the expert users. The comparison shows that both tools have similar acceptation scores, but OWL VisMod presents better feelings regard ing user's perception tasks due to the visual analytics influence.
\end{abstract}

\section{Introduction}

Semantic technologies are one of the fastest developing fields within the Information and Communication Technology sector and, as such, under constant examination by scientists and IT pro fessionals (Janev \& Vranes, 2011). Semantic, from the Greek "sēmantikos", involves giving significance or meaning to words or symbols, enabling distinctions between the meanings of differ ent words or symbols. Semantic technologies are based on ontolo gies (Fensel, 2002). Ontology formalizes knowledge meaning and facilitates the search for contents and information (Jiang \& Tan, 2009). The main objective of ontologies is to establish ontological agreements, which serve as the basis for communication between either human or software agents, hence, reducing language ambi guity and knowledge differences between agents, which may lead to errors, misunderstandings and inefficiencies (Blanco, Lasheras, Fernández Medina, Valencia García, \& Toval, 2011).

Now, semantic technology research relies on a number of key methodologies such as knowledge representation languages or reasoning algorithms (Hitzler \& Janowicz, 2011). The application of ontologies for expressing semantics of data does not restrict any longer exclusively on semantic web or semantic web services (Vrba, Radakovič, Obitko, \& Mařík, 2011).

According to Breslin, O’Sullivan, Passant, and Vasiliu (2010), industry has begun to watch developments with interest and a number of large companies have started to experiment with Semantic technologies to ascertain if these new technologies can be leveraged to add more value for their customers or internally

\footnotetext{
* Corresponding author.

E-mail address: ricardo.colomo@uc3m.es (R. Colomo-Palacios).
}

within the company, while there are already several offers of ven dors of Semantic solutions on the market. Due to this expansion several fields has been affected by semantics and many solutions and initiatives have been developed. Software Engineering is one of them. As a result of this there are many initiatives reported in the literature that employ semantic technologies in aspects like requirements (Chicaiza, López, Piedra, Martínez, \& Tovar, 2010), analysis (Tappolet, Kiefer, \& Bernstein, 2010, modeling (Gallardo, Molina, Bravo, Redondo, \& Collazos, 2011; Martinho, Varajao, \& Domingos, 2010; Sicilia, Sicilia, Sánchez Alonso, García Barrioca nal, \& Pontikaki, 2009), teaming (Soto Acosta, Casado Lumbreras, \& Cabezas Isla, 2010; Valencia García, García Sánchez, Castellanos Nieves, Fernández Breis, \& Toval, 2010), cooperative building (Tacla, Freddo, Paraiso, Ramos, \& Sato, 2011), software metrics (García Crespo, Colomo Palacios, Gómez Berbís, \& Mencke, 2009), reuse (Shiva \& Shala, 2008) or quality management (García et al., 2010) to cite some of the most relevant and recent cases.

Ontologies represent one of the most common representations of the semantic technologies (García Peñalvo, García, \& Therón, 2011). There is a research field called Ontology Engineering, which provides the mechanisms to manage the life cycle of them. The Ontology Engineering has been described as an investigation meth odology that provides the rational design of a knowledge base (Mizoguchi, 2004). It also provides the principles for the set of activities and processes that cover the life cycle of ontologies. The main of these processes are the creation, management, analy sis and reuse of ontologies.

As well as the processes, the Ontology Engineering also covers other aspects such as metrics, methodologies and the diverse tools for creating, editing and visualizing ontologies. Most of these ontology editors and tools are based on the use of simple visualiza 
tions, having diverse problems, as has been widely documented (e.g. García, García Peñalvo, \& Therón, 2011; García, Therón, \& García Peñalvo, 2011). These problems are mainly the occlusion of visual elements, the overcrowded visualizations, a lack of robust interaction techniques and a poor implementation of the visual expressivity, a concept defined as the number of visual variables used for enriching visualizations (Ware, 2004).

A solution to these visualization problems is the use of Visual Analytics techniques. Visual Analytics is a multidisciplinary research field focused on the development of diverse analytical reasoning techniques, visual representations and interaction tech niques, combined with a set of data representations and transfor mations. It has been more formally defined as: Visual analytics is the science of analytical reasoning facilitated by interactive visual interfaces (Thomas \& Cook, 2005).

In the Visual Analytics field, the user represents the main aspect in the process of analysis. He develops the analysis and the tools support this process. It is crucial the development of robust tools and visual and interactive techniques that support this analysis. This field is based on the use of the human cognitive capacities en riched with the currently computer capabilities. The result is a set of robust tools that the user can use to analyze information, and based on this analysis, first, to get knowledge from the data model and second, to take decisions or to execute diverse actions.

Visual analytics has been used in diverse research domains, such as bioinformatics (Baehrecke, Dang, Babaria, \& Shneiderman, 2004), Geography (Andrienko et al., 2007) or Medicine (Tominski, Schulze Wollgast, \& Schumann, 2008). Moreover, the industry it is also taking advantage in diverse fields such as databases (Shneiderman, 2008), Software engineering (Isenberg \& Fisher, 2009; Telea \& Voinea, 2009) or the pharmacy (Saffer, Burnett, Chen, \& van der Spek, 2004). Nevertheless, there is no any anteced ent of the use of visual analytics in the field of ontological engi neering (e.g. Gómez Pérez, Fernández, \& Corcho, 2003).

The advantages of using a Visual Analytics approach to develop the Ontological Engineering are diverse. The first advantage is that the use of robust visualization techniques, let to discover new knowledge of the ontologies, specially, during an analysis phase for reusing.

A second advantage is that the visual modeling process of creat ing ontologies becomes easier than the use of traditional ontolo gies editors based on widgets such as comboboxes, textfields, etc. Without any doubt, the use of visualizations improves the cogni tive process to analyze an ontological model.

This paper is focused on providing a validation of the OWL Vis Mod tool, which aims to contribute to the development of Ontolog ical Engineering, the branch of knowledge engineering that exploits the formal principles to build ontologies. The main pur pose behind OWL VisMod is to provide users with a tool to support the development, creation, management, maintenance and reus ability of OWL ontologies for knowledge based systems (García, García Peñalvo, \& Therón, 2010a; García, García Peñalvo, \& Therón, 2010b). The usability of OWL VisMod has been evaluated by means of an empirical study, with good results (García, García Peñalvo, Therón, \& Ordóñez de Pablos, 2011).

The paper consists of four sections and is structured as follows. Section 2 reviews the relevant literature about the field of study of OWL VisMod. Section 3 describes the tool paying attention to its architecture and main features. Section 4 describes the evaluation process carried out. Finally, the paper ends with a discussion of re search findings, limitations and concluding remarks.

\section{Literature review}

The main processes involved in the life cycle of ontologies are the creation, maintenance, analysis and reuse. The creation process consists of activities and workflows that have been defined in di verse methodologies. Uschold and King (1995) proposed one of the first methodologies specially focused on the creation process, called Knowledge Engineering Methodology (KEM). This proposal describes some of the most important tasks, involved in the pro cess of the creation of ontologies. Fig. 1 illustrates the most impor tant activities defined in the KEM Methodology. It starts with the definition and conceptualization of the domain, followed by an analysis phase in order to reuse existing ontologies in the model that is being built. Then, the formal specification of the ontology includes the definition of the taxonomy of concepts, the attributes and relations. Once the ontology has been built, the next phase in volves the creation of the individuals or instances that populate the ontology, to finally conclude with the evaluation and documenta tion processes.

Another relevant methodology that has been taken as base for future proposals is Methontology (e.g. Fernández López, Gómez Pérez, \& Juristo 1997). Methontology covers the whole life cycle of ontologies, and includes a tool called WebODE that sup ports all the activities defined on it.

Methontology is focused on the development of ontologies from the level of knowledge, through an approach close to the tradi tional cascade process defined in the Software Engineering field. This proposal defines four phases to build an ontology: the first phase is the definition of the reach and the granularity, the second phase is the conceptualization of the domain, the third phase is the implementation of the ontology in a language such as RDF or OWL. Finally, the fourth phase is the evaluation of the ontology.

DOGMA (Development of Ontology Guided Methodology Ap proach) is a framework for developing the Ontology Engineering in a very formal manner (Jarrar \& Meersman, 2002). The philoso phy behind DOGMA is the reuse of ontologies, due to they are con sidered as scalable and shared resources that let to reuse the knowledge (Jarrar \& Meersman, 2009). The reuse of the ontologies is due to the methodology proposes the definition of diverse levels of abstraction, starting from an upper level with very general con cepts, that can let these models to be reused in diverse domains.

Apart from the methodologies, the Ontology Engineering also requires tools that support all the activities defined in the pro cesses. Diverse tools have been designed (Suresh, Kumar, Prakash, \& Rizvi, 2008), nevertheless, all these proposals do not support methodologies. In contrast, they are independent proposals, except for Methontology and DOGMA that have implemented specific tools that support the activities defined.

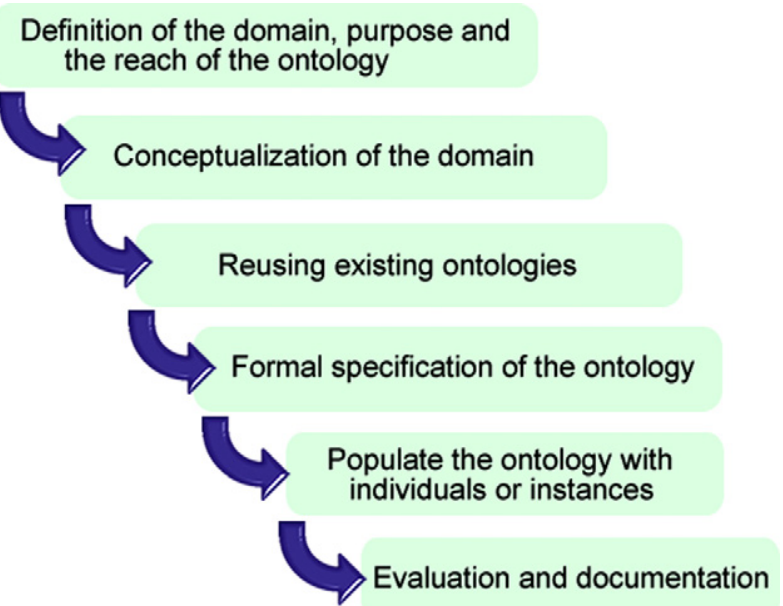

Fig. 1. Six defined phases in the methodology Knowledge Engineering Methodology (KEM). 


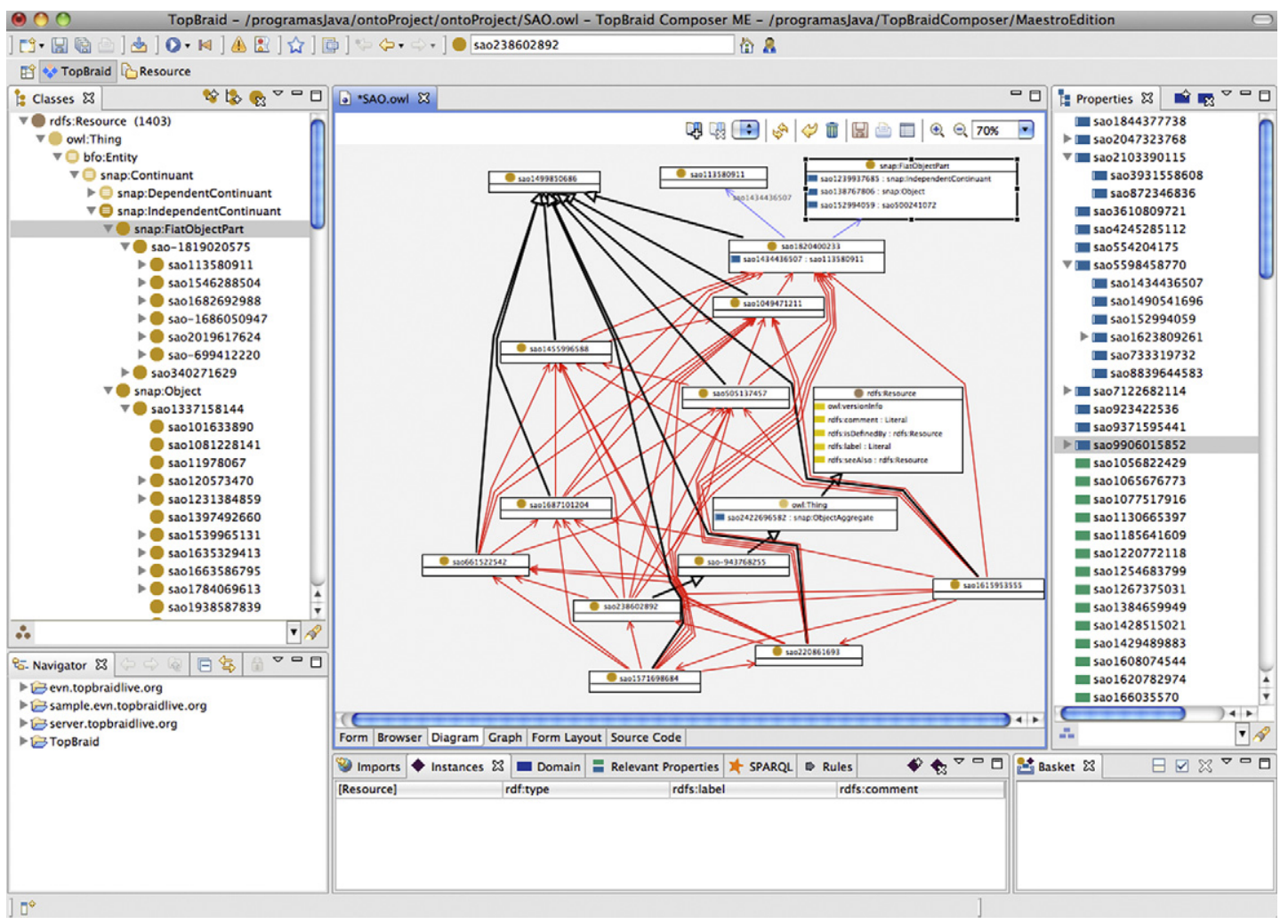

Fig. 2. TopBraid Composer uses a UML-based visualization to represent both the hierarchy and the relations among classes.

Diverse commercial tools have been proposed for modeling ontologies. The most important currently are: SemanticWorks, TopBraid Composer and OntoStudio. Some of these tools offer a free version with reduced funcionality. There are other free to use tools to model an edit ontologies such as NeOn Toolkit (Haase, Lewen, Studer, \& Erdmann, 2008), OntoEdit (Sure, Angele, \& Staab, 2002), HOZO (Kozaki, Kitamura, \& Mizoguchi, 2005; Sunagawa, Ko zaki, Kitamura, \& Mizoguchi, 2005), but Protégé (Gennari et al., 2003 ) is the most widely used tool for editing ontologies.

SemanticWorks ${ }^{1}$ is a commercial tool designed to edit RDF doc uments in a GUI and check its sintaxis, as well as design RDF schema and OWL ontologies using a graphical design View, based on a con ceptual map approach. It checks the syntaxes and semantics of ontologies, using a graph modeler based on the use of conceptual maps.

The European Union has visualized the potential of the develop ment of the diverse semantic technologies, and has supported re search in this direction. The project $\mathrm{NeOn}^{2}$ can be the most important proposal in this area. Its main goal is to manage multiple ontologies in a specific context, that are created as result of a collab oration of diverse entities, and can be dynamic and under evolution.

As a result of this research project, diverse tools and applica tions ontology based have been released (e.g. Suárez Figueroa et al., 2007; Villalón Terrazas, Ramínez, Suárez Figueroa, \& Gómez Pérez, 2011). One of the most important tools is an envi ronment for developing the Ontology Engineering called NeOn Toolkit (Haase et al., 2008). This robust platform is open to new developments of Eclipse plugins that can be added to the toolkit.

TopBraid Composer ${ }^{3}$ illustrated on Fig. 2 is an enterprise class modeling environment for developing Semantic Web ontologies

\footnotetext{
${ }^{1}$ http://www.altova.com/semanticworks/owl-editor.html.

${ }^{2}$ http://www.neon-project.org/.

3 http://www.topquadrant.com/.
}

and building semantic applications. There are three available ver sions: a Free Edition, Standard Edition and Maestro Edition. TopBraid Composer is a UML based modeling plug in eclipse, part of the Top Braid Suite. We tested using TopBraid Composer Free Edition version 3.3.0 which does not support the UML representation that is pro vided only with paid versions. TopBraid Composer is a fully Pro tege based tool that performs the most common operations over ontologies, such as: inference, consistency checking, and the inclu sion of SPARQL query engine.

Protégé (Gennari et al., 2003) is a free, open source ontology editor and knowledge based framework. Protégé includes diverse plugins developed and maintained by the community. One of these plug ins is OWLViz, a graph based visualization that represents classes, properties, hierarchy, and the classical tree of hierarchies view. Classes are represented as nodes in the graph, while proper ties are represented as edges connecting nodes, where the edges represent "is a" relationships (hierarchy).

Jambalaya (Storey et al., 2001) is another plug in intended to visualize OWL ontologies with Protégé. It is a visualization tool not provided with modeling capabilities. Jambalaya is a complete plug in that visually represents the components of the ontology and its relationships divided into two views. Each view can be dis played using one of six different layouts: grid, radial, spring, sugiy ama, tree and treemap. This tool offers a great variety of configuration options hiding components, changing colors and shapes and filtering data. Although Jambalaya represents a very good tool to visualize an ontology, the scalability is the main disad vantage due to the fact that large graph visualizations are well known to become cluttered.

Katifori, Halatsis, Lepouras, Vassilakis, and Giannopoulou (2007) provided with a classification of the diverse tools for visual izing ontologies. They defined six categories according to the dif ferent characteristics of the presentation, interaction, technique, functionality supported or visualization dimensions. Nevertheless 


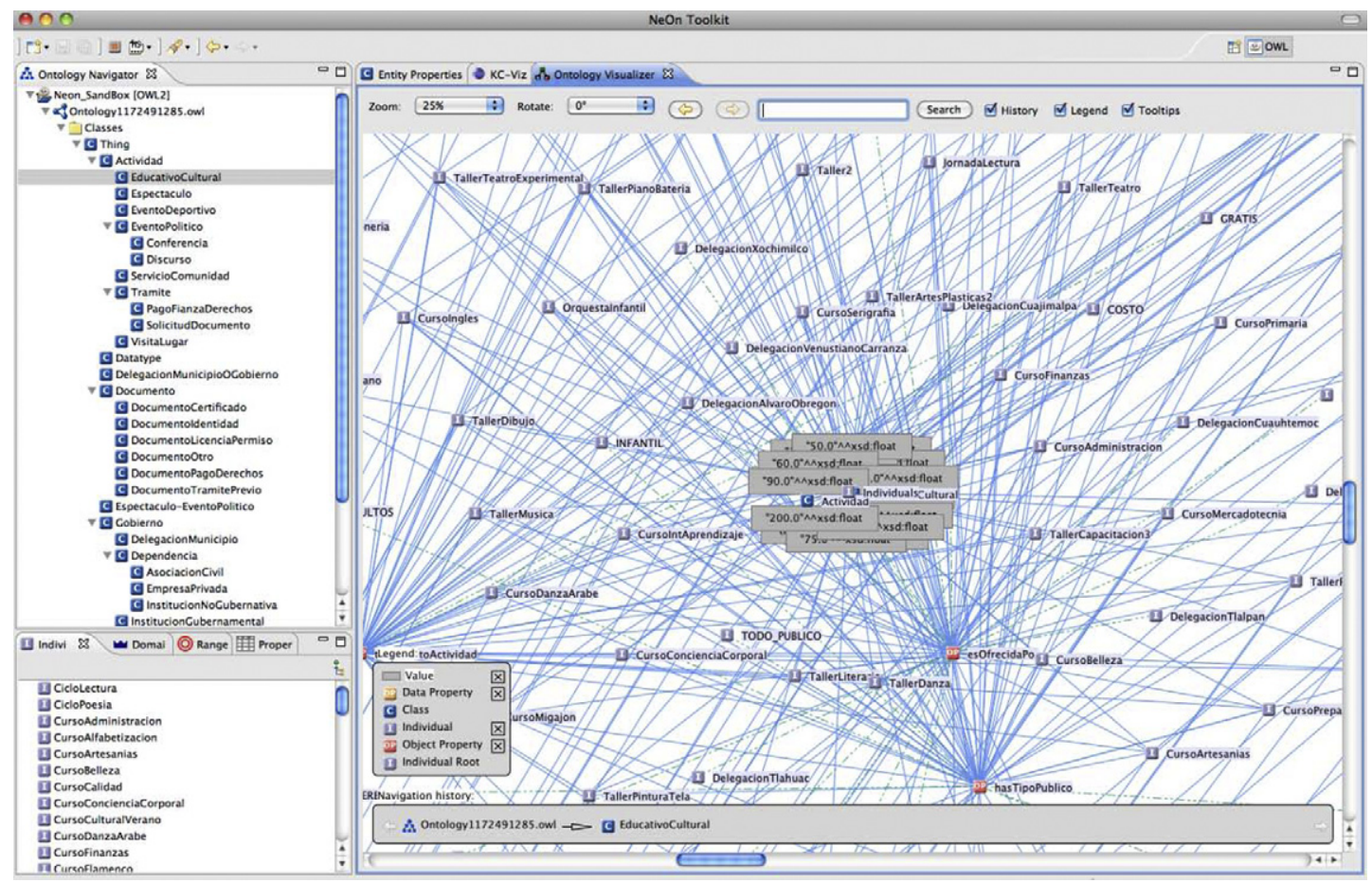

Fig. 3. The NeOn Toolkit illustrates the problems of oclussion and overcrowding of visual elements.

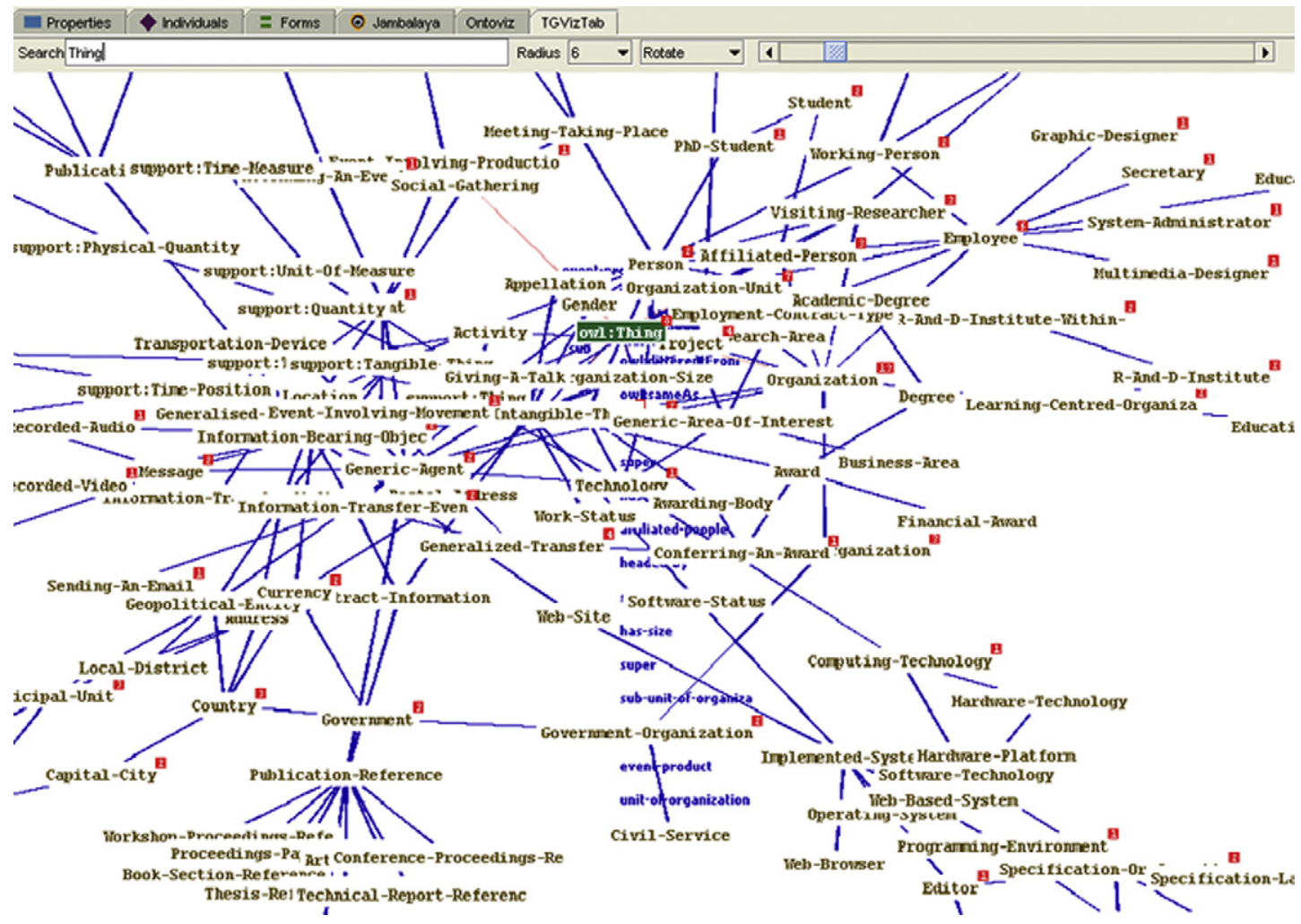

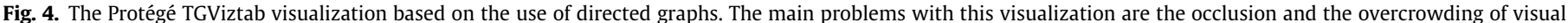
elements.

most of the tools fall in more than one category. There are two groups not included in this category; the first group is based on those tools that use UML notation to model ontologies, and the sec ond group is formed by those tools based on the use of conceptual maps.
These eight groups are: indented lists, node link (graphs) and trees, zoomables, space filling, focus + context or distortion, 3D information landscapes, the UML based and the conceptual maps based. Basically, it can be distinguished two main modeling approaches for representing the relationships among classes: the 


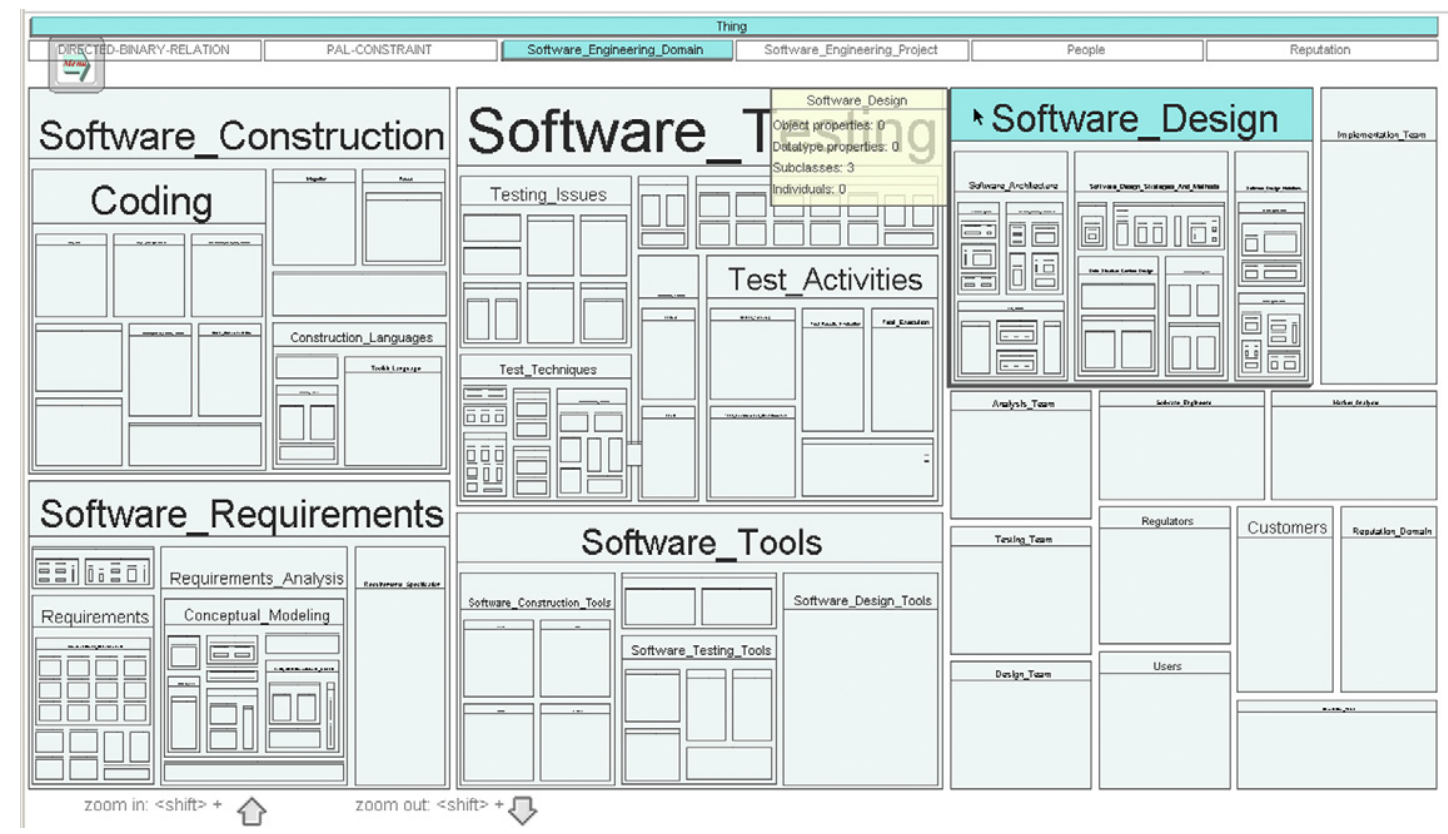

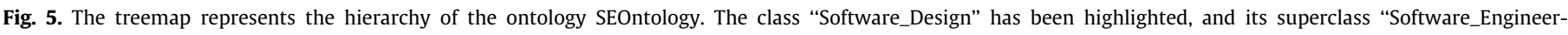
ing_domain" is also highlighted.

first one using the well known graph theory (like Protégé and SemanticWorks) and the second approach using UML diagrams, such as in the Object Oriented approach (TopBraid Composer).

All the tools based on the use of graphs (node link) share the same problems. The first is the lack of a layout, and the majority of the time the user has to manually move the visual elements to organize them. The second problem is the scalability. It is well known that graphs are not good to represent a large amount of ele ments; these problems are illustrated on Figs. 3 and 4. The tools based on UML practically have the same problems which are illus trated on Fig. 2, even when this figure solely visualizes less than twenty classes.

As a result of an analysis of the current diverse tools, we have identified the main problems, including: the symbol redundancy (SemanticWorks), overcrowding of visual elements that difficult the understanding of visualizations, such as those based on direc ted graphs or UML. This problem is caused due to the majority of the tools saturating the visualizations and putting together the tax onomy with relationships. Therein the user gets easily confused and lost navigating the visualization. Another detected problem is the lack of layout, in the case of graphs and UML diagrams. A lack of a layout makes it difficult to find elements, and create a concep tual map of the knowledge base that is represented.

Fig. 3 illustrates the main problems that share the most of the visualizations tool. First, due to most of them use basic visualiza tion techniques such as directed graphs, as shown on Fig. 3. One of the problems is that visualizations display all the information in the same views, such as properties, classes or individuals. This strategy causes that as can be seen on Fig. 3, the visual elements overlap others, the edges cannot be followed, and the visualization becomes completely overcrowded.

Most of the tools share the same problems, due to as can be seen on Fig. 2 with TopBraid Composer and Fig. 3 with NeOn Tool kit, most of them have decided to follow the Protégé's approach, sharing its same problems. Fig. 4 shows the Protégé TGVizTab view, which is based on the use of directed graphs. It can be seen that the three tools described, share exactly the same problematic. Moreover, other tools described in García et al. (2011) also share the same problematic.

\section{The tool}

The analysis started with the loading of the ontology ${ }^{4}$ in OWL VisMod. Then, the first activity consisted on the navigation of the taxonomy of concepts, to detect those related to software safety. The taxonomy of concepts is defined in OWL VisMod using two visu alization techniques: the treemap and the hierarchical tree.

The first technique is the treemap (Johnson \& Shneiderman, 1991), a widely used technique for representing especially, large hierarchies (Bederson, Shneiderman, \& Wattenberg, 2002). This visualization technique is based on the efficient use of the whole available visual space in the dimensional plane. It is based on the use of two dimensionally squared maps, where the lower levels are represented as internal squares located inside the higher level maps.

Fig. 5 shows a treemap view representing the taxonomy of the SEOntology. This analysis is focused on those classes related to software security, so the class called "Software_Design" has been highlighted in order to analyze its subclasses. The total of classes in the ontology is 365 , with 180 datatype properties, and $129 \mathrm{ob}$ ject properties.

The main classes are subclasses of the class "Software_Engi neering_Domain”, and these classes are: Software_Design (high lighted in Fig. 5), Software Testing, Software_Construction, Software_Tools and Software_Requirements. All these classes re lated to the different processes involved in a software developing process are shown in the Fig. 5. These classes represent the main aspects involved in the life cycle of a software project.

The second visualization technique that is used to analyze the taxonomy of concepts is the hierarchical tree. This visualization is a complementary view of the treemap, it uses the representation model of edges that connect the nodes, representing the elements (Tominski, Abello, Van Ham, \& Schumann, 2006).

The analysis to the taxonomy of concepts, let to identify that there are no specific security related concepts in this ontology. Di verse aspects related to the software engineering project have been

\footnotetext{
${ }^{4}$ http://www.seontology.org/permit_on/src/genericOnto.owl.
} 


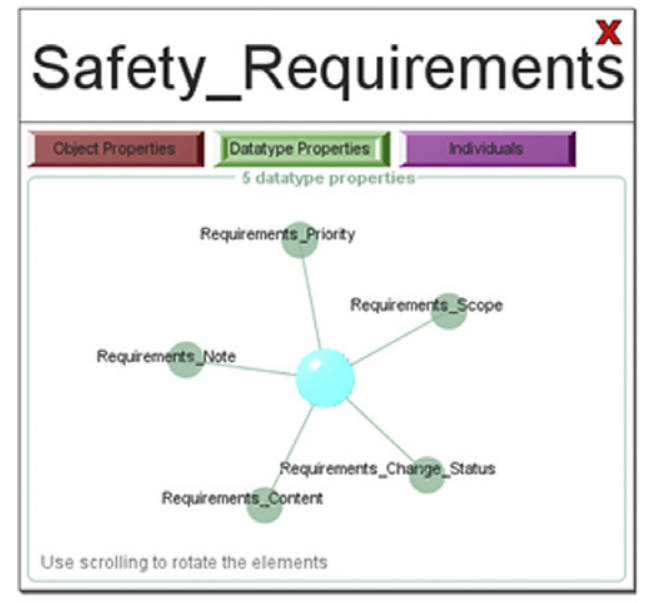

(a)

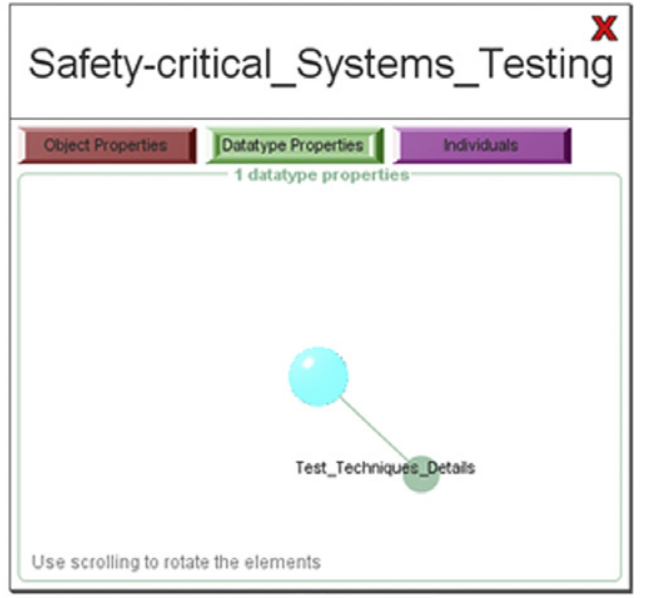

(b)

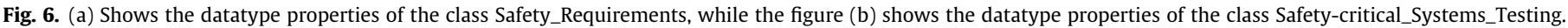

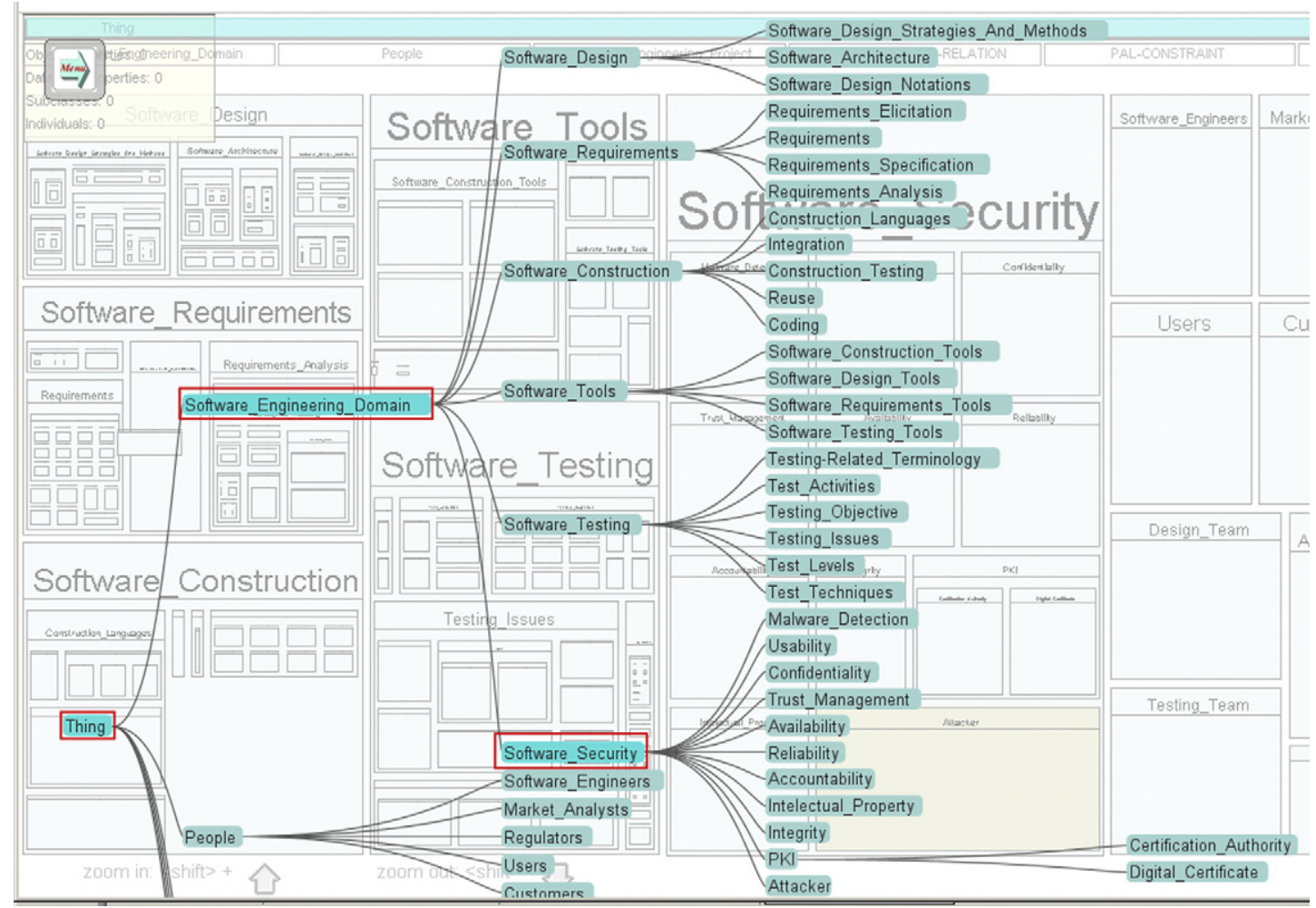

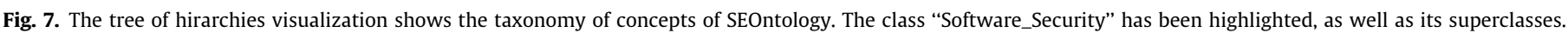

defined, nevertheless, the problem of security and the safety software has not been considered in this ontological model. There are just two general concepts involved with security: the class "Safety_Requirements" and the class "Safety critical_Systems_ Testing". Nevertheless, neither of them has defined object proper ties nor subclasses. Fig. 6(a) shows the datatype properties of the class Safety Requirements, in this case five properties. On the other hand the class Safety critical Systems Testing has only one datatype property defined.

This semantic zoom representation is based on a UML like view, having an internal graph with a radial layout (García et al., 2011). Colors are used to indicate the type of the property or individuals. More specifically, the red is used to represent object properties, the green is used to represent the datatype properties, while the purple is used to represent the individuals of a class.
Fig. 6(a) and (b) use the semantic zoom visualization technique to display the details of a specific class in the ontology. This tech nique is based on showing details according to the user's needs, changing the type and the meaning of the displayed information. Its main advantage is that the global context can be remained, while a detailed view of a certain element is shown (Herman, Mel ancon, \& Marshall, 2000).

OWL VisMod has implemented a visualization technique to represent the global coupling of an ontology (García et al., 2011). This visualization shown on Fig. 8 is based on a radial layout of the coupled classes in the ontology. Relations among classes also called coupling relationships are defined using Bezier curves in the same way, called Hierarchical Edge Bundles (Holten, 2006). This type of edges can be "tensed" to get more clear visualizations, avoiding the occlusion of edges. The use of a color varying from a 


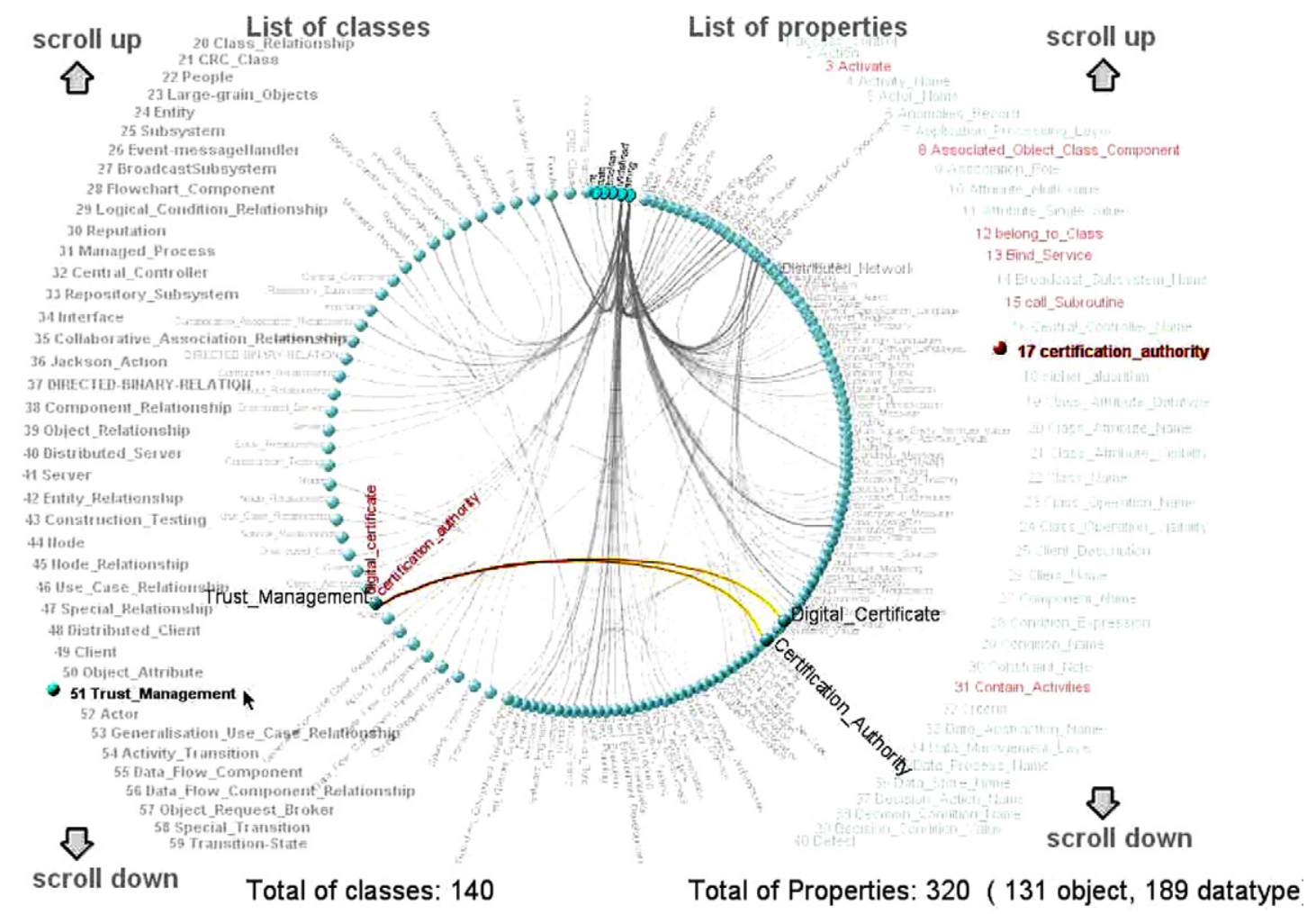


"Digital_Certificate" and "Certification_Authority".

tone to other, indicates the direction of the property, avoiding the use of arrowheads that overcrowd the visualization with elements. For the specific case of OWL VisMod, the object properties are rep resented with a curve varying from red to yellow, while the data type properties are represented with a curve varying from green to yellow.

Another visualization technique that has been implemented is the coupling of a specific class (García et al., 2010b), according to the coupling metrics defined in (García et al., 2010a). This visuali zation has been implemented in order to represent the semantic meaning of the coupling among classes. This coupling is inter preted as a relationship between two classes, where this relation ship is defined as a mathematical function, having a class in its domain, and a class in its range. This interpretation of the semantic will be explained in detail with an example, in the next section.

According to the result of this analysis, the first action that should be taken, is the creation of diverse concepts related to safety mechanisms, in order to enrich this ontological model. Then, for each new class created, diverse properties should be defined, to finally populate the ontological model with instances of the class. These activities are described in detail in the following section.

\section{Tool evaluation}

With the aim of getting feedback concerning the tool compared with well known solutions, an evaluation was carried out as de scribed in the subsequent paragraphs.

\subsection{Experimental design}

The evaluation of the system consists in the performance of a set of tasks by a set of subjects. This set of tasks will be performed using a Domain Ontology devoted to Software Engineering, in this case Software engineering ontology (SEOntology), and performed using two different tools. The first set of tasks will be performed using OWL VisMod and the second one using Protégé. After these tasks, all users are asked to answer a questionnaire. The final aim is to compare the results of both questionnaires in order to set the validity of OWL VisMod compared with a recognized tool like Protégé.

The task is divided in several steps described as follows:

1. Load SEOntology.

2. Navigate the taxonomy looking for specific concepts. In this case, related to software safety.

3. Analysis of Safety_Requirements and Safety critical_Sys tems_Testing classes (both related to software safety), checking that both are defined as isolated classes.

4. Define new classes as follows:

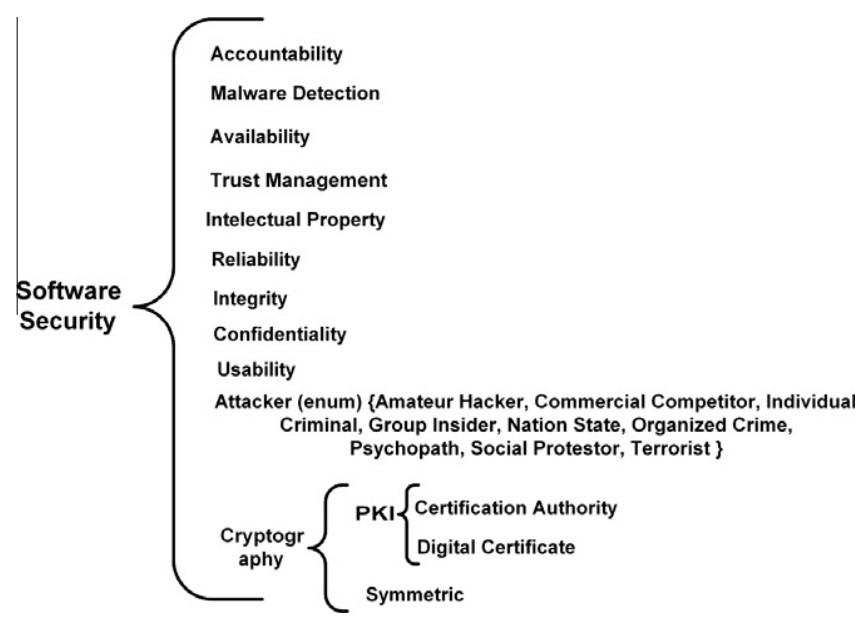


5. Define properties as follows:

\begin{tabular}{|c|c|c|}
\hline Class & Type & Name \\
\hline Malware_Detection & $\begin{array}{l}\text { datatype } \\
\text { (String) }\end{array}$ & detection_mechanism \\
\hline Malware_Detection & $\begin{array}{l}\text { datatype } \\
\text { (String) }\end{array}$ & malware_name \\
\hline Intelectual_Property & $\begin{array}{l}\text { datatype } \\
\text { (boolean, } \\
\text { functional) }\end{array}$ & Registered \\
\hline Trust_Management & $\begin{array}{l}\text { Object } \\
\text { (Certification } \\
\text { Authority) }\end{array}$ & certification_authority \\
\hline Trust_Management & $\begin{array}{l}\text { datatype } \\
\text { (boolean, } \\
\text { functional) }\end{array}$ & uses_certificate \\
\hline Trust Management & $\begin{array}{l}\text { Object(Digital } \\
\text { Certificate) }\end{array}$ & digital_certificate \\
\hline Reliability & $\begin{array}{l}\text { datatype } \\
\text { (String) }\end{array}$ & identity_management \\
\hline Usability & $\begin{array}{l}\text { datatype } \\
\text { (String) }\end{array}$ & $\log$ \\
\hline Confidenciality & $\begin{array}{l}\text { datatype } \\
\text { (String) }\end{array}$ & access_control \\
\hline Confidenciality & $\begin{array}{l}\text { object } \\
\text { (Symmetric) }\end{array}$ & cipher_algorithm \\
\hline Integrity & $\begin{array}{l}\text { datatype } \\
\text { (String) }\end{array}$ & hash_algorithm \\
\hline
\end{tabular}

6. Define instances as follows:

\begin{tabular}{ll}
\hline Classes & Instances \\
\hline Certification_Authority & $\begin{array}{l}\text { VerySign, CERES, GVA } \\
\text { Digital_Certificate }\end{array}$ \\
VerySignCertificate, \\
Malware_detection & $\begin{array}{l}\text { CERES_Certificate, GVA_Certificate } \\
\text { detection_mechanism = static } \\
\text { analysis based } \\
\text { detection_mechanism = code graphs } \\
\text { based } \\
\text { DES, 3DES, AES, Blowfish }\end{array}$ \\
\hline
\end{tabular}

\section{Save the ontology}

Once subjects performed the tasks they were asked to answer a questionnaire. This questionnaire consisted on a set of questions that evaluate the performance of OWL VisMod and Protégé using the same set of questions by means of a Likert scale ( 110 points with anchors from very poor to very satisfactory):

- Rate taxonomic visualization of the classes that this tool provides.

- Rate the visual analytics process of the tool.

- Rate the ontology modeling workflow.

- Rate the user experience of the tool.

- Rate the global performance of the tool.

A pilot application was made prior to the final implementation of the questionnaire. The sample for this pilot implementation was composed by three semantic technologies experts. The objective of this pilot study was the improvement and assurance of the associ ated documentation. This resulted in several changes in formats and tables in the wording of some texts.
Data collection was conducted through a questionnaire that ob tained information from the sample. All questionnaires were filled out by subjects with the assistance of at least one researcher. Ques tionnaires were answered on printed copies and subsequently coded in the statistical analysis tool GNU R.

\subsection{Sample}

The study was carried out over a period of two weeks. Partici pants were obtained from those who responded positively to a per sonal invitation, the sample consisted of 21 subjects, 4 women and 17 men. The average age was 27.9. In average, subjects have 2.3 years of experience in the field of semantic technologies.

\subsection{Threats to validity}

In this study internal or external validity threats are present. With respect to the first, the respondents may not have a compa rable level of knowledge or expertise. However, sample was chosen because of their expertise and experience, authors made sure that experts possessed a comparable level of knowledge and expertise.

The Fig. 7 depicts the tree visualization after the creation of the concepts related to security. The class Software Security has been highlighted in a red colored rectangle, as well as its superclass Soft ware Engineering Domain. This class is defined as an upper class for diverse concepts related to the security of the information, such as Confidentiality, Usability, Availability, Reliability or Trust Manage ment among others, shown in the Fig. 7. In total, fourteen new con cepts have been created to the ontological model, as previously has been described.

Once the new concepts have been created in the ontology, the next phase is the definition of the relations among classes. Fig. 8 shows the relations of the class Trust Management with the classes Digital Certificate and Certification Authority. These relationships indicate that in order to have a trusty relation between two parts, there is a need of having a digital certificate validated by a certifi cation authority.

The visualization technique shown in Fig. 8, highlights the fo cused class (Trust Management) as well as the coupled classes (Dig ital Certificate and Certification Authority), while the rest of classes are blurred, in order to implement a Focus + Context, which re mains the global context and highlights the focused elements.

The names of the coupled classes and the relationships are listed using a circular list that rotates to display the next group of elements. This interaction lets the user to navigate over all the values, without being important the total number of elements.

Fig. 8 has shown the global coupling of the whole ontology, where all the coupled classes are shown in a global context. More over, the user can be interested in the coupling of a specific class, such as the classes Digital Certificate and Trust Management, de picted on Fig. 9(a) and (b). These classes are related or coupled, by means of the relation called digital certificate.

This coupling relationship has been shown on Fig. 8, using a gen eral view. The interaction with the user includes the semantic zoom visualization shown on Fig. 9(a) and (b), in response of a user selec tion of the class. Fig. 8 shows this relationship in the sense going from the class Trust Management (in the domain) to the class Digi tal Certificate (in the range). This direction is indicated with the col or of the edge, from the red to the yellow. Moreover, this relation is also depicted on Fig. 9(a) and (b), where the class Trust Manage ment remains to the left side of the class Digital Certificate, indicat ing the manner that the relation has to be interpreted.

These Figures represent three different views or perspectives of a coupling relationship. The first (Fig. 8) is a general view, the sec ond (Fig. 9(a)) is a semantic zoom view (detailed view) having as the selected element the class Digital Certificate, while the third 


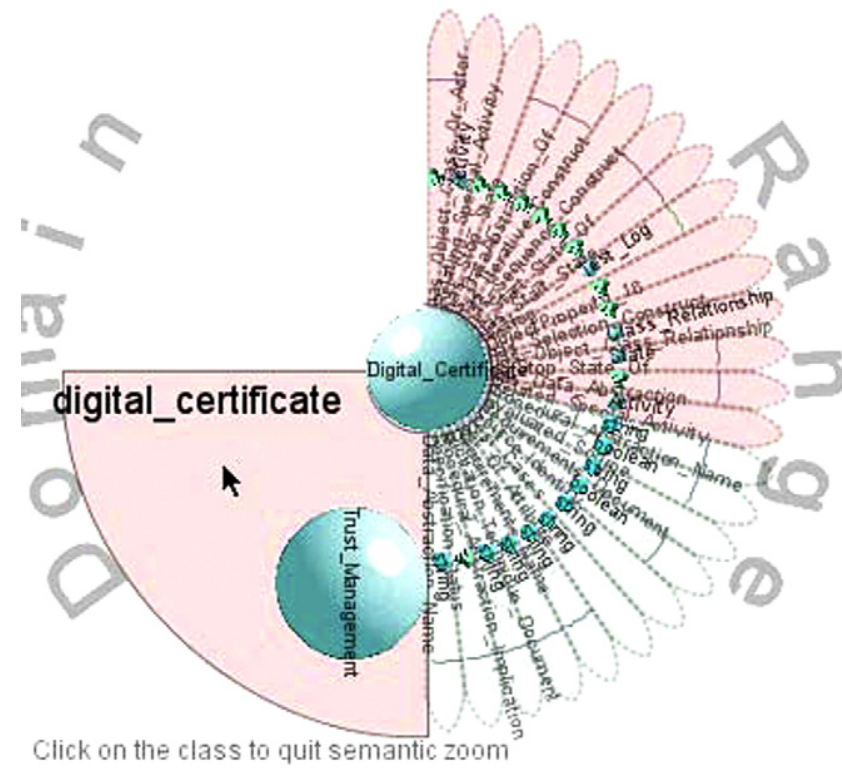

(a)

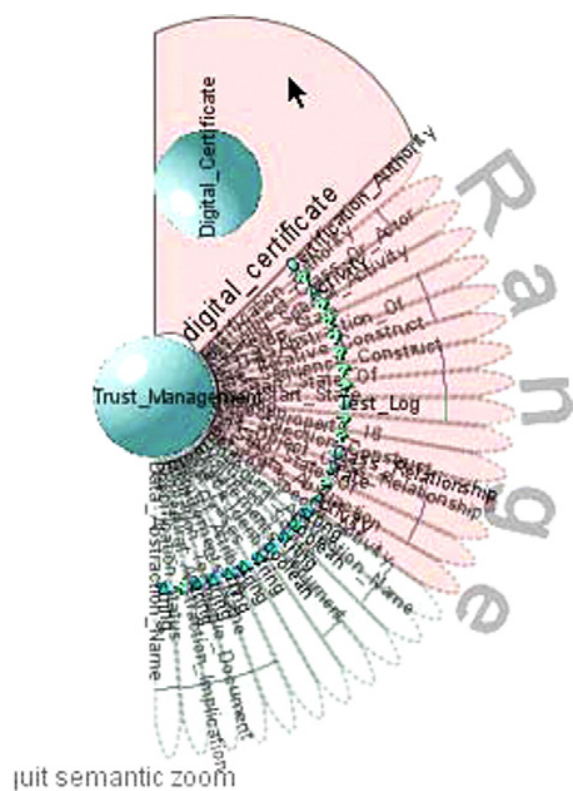

(b)

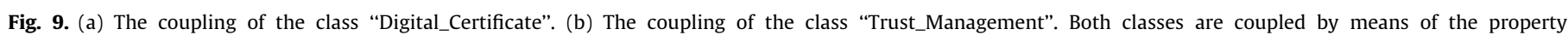
"digital_certificate", which indicates that a trusty relationship is performed by means of a Digital Certificate.

Table 1

Evaluation of statistical results.

\begin{tabular}{llllll}
\hline & OWL-VisMod & & & \multicolumn{2}{c}{ Protégé } \\
\cline { 2 - 3 } \cline { 5 - 6 } & Average & Standard deviation & & Average & Standard deviation \\
\hline Taxonomic visualization & 7.19 & 1.123 & 0.577 & 6.48 & 1.108 \\
Visual analytics process & 6.33 & 0.805 & 6.43 & 0.512 \\
Ontology modeling workflow & 7.38 & 0.669 & 6.38 & 1.121 \\
User experience & 7.05 & 1.108 & 7.00 & 0.973 \\
Global performance & 6.86 & & & 0.894 \\
\hline
\end{tabular}

one (Fig. 9(b)) is a semantic zoom but having as the selected ele ment the class Trust Management.

Regarding external validity, there are two possible threats. The first is the small number of respondents, which makes difficult the generalization of results. The second is the fact that the sample was not taken randomly. Future works will tackle both threats.

\section{Results and discussion}

Table 1 presents average and standard deviation of the re sponses offered by the subjects in relation to the questionnaire ap plied and the two groups of questions formulated.

Results show that, in general, punctuations are similar for both tools in every aspect. However, it is important to underline that in two factors, namely "Ontology modeling workflow" and "User experience" average values are quite higher in the case of OWL VisMod, presenting also in these cases lesser and moderate stan dard deviation values also.

In an attempt to verify whether results presented statistically significant differences, the statistical $t$ test (comparison of two means) was used to analyze if differences between the two groups existed. The level of statistical significance was set at 0.05 . The anal ysis was conducted for each factor. This analysis indicated that two variables present significant differences: User Experience $(t(42)=2.586, \quad p<.05)$ and Ontology modeling workflow $(t(42)=3.162, p<.05)$. In contrast none of the variables that ob tained higher values in Protégé than in OWL VisMod present signif icant differences: Global performance $(t(42)=-.460, p>.05)$ and Visual analytics process $(t(42)=-.849, p>.05)$. Finally, the variable Taxonomic visualization that obtained higher values for OWL Vis Mod does not present significant differences $(t(42)=.891, p>.05)$.

Results mean that the implementation of OWL VisMod may be considered a notable success. This tool is comparable in terms of global performance to a standard like Protégé and can provide good results in aspects like User Experience and Ontology Model ling Workflow.

\section{Conclusions}

The development of ontologies represents a crucial aspect in the Knowledge Engineering field. Developing an ontological model is very complicated task, independently if the model is built up from the scratch or reusing an existing ontology.

The ontology life cycle management has all the related issues about knowledge abstraction and modeling with the different notation and methods supported by ontology tools. All these pro cesses present a big gap between the conceptual models in the ex perts' brains and the ontological models.

OWL VisMod tool contributes to the development of Ontologi cal Engineering that exploits the formal principles to build ontolo gies and is based on Visual Analytics techniques to reduce this conceptual gap and close de cycle between the conceptual abstrac tions and models, allowing the interaction with visual models to 
extract new knowledge or a better understanding the models in or der to iterate in the ontology life cycle processes.

In this paper, OWL VisMod has been validated performing a set of conceptual modeling tasks using the SEOntology Software engi neering ontology. The tasks have been performed using OWL Vis Mod and also with Protégé, with the final aim of comparing the results in order to set the validity of OWL VisMod compared with a recognized tool like Protégé is in the Knowledge Engineering field.

Evaluation results show that both tools have similar scores in every aspect, but OWL VisMod presents better average values in the "Ontology modeling workflow" and "User experience", which it means the importance of a visual analytics in the human depend ing tasks of knowledge engineering processes. Given the irruption of Linked Data in the semantic technologies scenarios (Colomo Palacios, Sánchez Cervantes, Alor Hernández, \& Rodríguez González, 2012), future works are aimed to adapt OWL VisMod to Linked Data visualization.

\section{References}

Andrienko, G., Andrienko, N., Jankowsk, P., Keim, D., Kraak, M., MacEachren, A., et al (2007). Geovisual analytics for spatial decision support: Setting the research agenda. International Journal of Geography Information Science, 21(8), 839-857.

Baehrecke, E., Dang, N., Babaria, K., \& Shneiderman, B. (2004). Visualization and analysis of microarray and gene ontology data with treemaps. BMC Bioinformatics, 5(1), 12-14.

Bederson, B., Shneiderman, B., \& Wattenberg, M. (2002). Ordered and quantum treemaps: Making effective use of $2 \mathrm{~d}$ space to display hierarchies. ACM Transactions on Graphics, 21(4), 833-854.

Blanco, C., Lasheras, J., Fernández-Medina, E., Valencia-García, R. \& Toval, A. (2011). Basis for an integrated security ontology according to a systematic review of existing proposals. Computer Standards E' Interfaces, 33(4), 372-388.

Breslin, J. G., O'Sullivan, D., Passant, A., \& Vasiliu, L. (2010). Semantic Web computing in industry. Computers in Industry, 61(8), 729-741.

Chicaiza, J., López, J., Piedra, N., Martínez, O., \& Tovar, E. (2010). Usage of social and semantic web technologies to design a searching architecture for software requirement artifacts. IET Software, 4(6), 407-417.

Colomo-Palacios, R., Sánchez-Cervantes, J. L., Alor-Hernández, G., \& RodríguezGonzález, A. (2012). Linked Data: Perspectives for IT professionals. International Journal of Human Capital and Information Technology Professionals, 3(3), 1-15.

Fensel, D. (2002). Ontologies: A silver bullet for knowledge management and electronic commerce. Berlin, Germany: Springer-Verlag.

Fernández-López, M., Gómez-Pérez, A., \& Juristo, N. (1997). Methontology: From ontological art towards ontological engineering. In Proceedings of the ontological engineering, AAAI-97. Spring symposium series (Vol. 1, pp. 33-40).

Gallardo, J., Molina, A. I., Bravo, C., Redondo, M. A., \& Collazos, C. A. (2011). An ontological conceptualization approach for awareness in domain-independent collaborative modeling systems: Application to a model-driven development method. Expert Systems with Applications, 38(2), 1099-1118.

García, J., García-Peñalvo, F., \& Therón, R. (2010a), Defining coupling metrics among classes in an OWL ontology. In 23rd International conference on industria engineering and other applications of applied intelligent systems, trends in applied intelligent systems, IEA/AIE 2010. Springer LNAI (Vol. 6097, pp. 12-17).

García, J., García-Peñalvo, F., \& Therón, R. (2010b). Visualising semantic coupling among entities in an owl ontology. In Proceedings of ONTOSE 2010, Ontology, conceptualization and epistemology for information systems, software engineering and service science (Vol. 62, Issue 3, pp. 90-106). Springer.

García, J., García-Peñalvo, F., \& Therón, R. (2011), Modelling relationships among classes as semantic coupling in owl ontologies. In Proceedings of the 2011 international conference on information and knowledge engineering, IKE 2011 (Vol. 1, pp. 22-28).

García, J., Therón, R., \& García-Peñalvo, F. (2011), Semantic zoom: A details on demand visualisation technique for modelling owl ontologies. In 9th International conference on practical applications of agents and multiagent systems, highlights in practical applications of agents and multiagent systems AISC'89 (pp. 85-92).

García, J., García-Peñalvo, F., Therón, R., \& Ordóñez de Pablos, P. (2011). Usability evaluation of a visual modelling tool for owl ontologies. Journal of Universal Computer Science, 17(9), 1299-1313.

García, R., Gil, R., Gimeno, J. M., Granollers, T., López, J. M., Oliva, M., et al. (2010). A Semantic Wiki for quality management in software development projects. IET Software, 4(6), 386-395.

García-Crespo, A., Colomo-Palacios, R., Gómez-Berbís, J. M., \& Mencke, M. (2009). BMR: Benchmarking metrics recommender for personnel issues in software development projects. International Journal of Computational Intelligence Systems, 2(3), 257-267.

García-Peñalvo, F., García, J., \& Therón, R. (2011). Analysis of the owl ontologies: A survey. Scientific Research and Essays, 6(20), 4318-4329.
Gennari, J., Musen, M., Fergerson, R., Grosso, W., Crubezy, M., Eriksson, H., et al. (2003). The evolution of protege: An environment for knowledge-based systems development. Stanford Medical Informatics, 58(1), 89-123.

Gómez-Pérez, A., Fernández, M., \& Corcho, O. (2003). Ontological engineering. Springer-Verlag.

Haase, P., Lewen, H., Studer, R., \& Erdmann, M. (2008). The neon ontology engineering toolkit. In Proceedings of the 17th international world wide web conference.

Herman, I., Melancon, G., \& Marshall, S. (2000). Graph visualization and navigation in information visualisation: A survey. IEEE Transactions on Visualisation and Computer Graphics, 6(1), 24-43.

Hitzler, P., \& Janowicz, K. (2011). Semantic Web tools and system. Semantic Web, $2(1), 1-2$.

Holten, D. (2006). Hierarchical edge bundles: Visualization of adjacency relations in hierarchical data. IEEE Transactions on Visualization and Computer Graphics, 12(5), 741-748.

Isenberg, P., \& Fisher, D. (2009). Collaborative brushing and linking for co-located visual analytics of document collections. Computer Graphics Forum, 28(3), 1031-1038.

Janev, V., \& Vranes, S. (2011). Applicability assessment of Semantic Web technologies. Information Processing and Management, 47(4), 507-517.

Jarrar, M., \& Meersman, R. (2002). Formal ontology engineering in the dogma approach. Proceedings of the international conference on ontologies, databases and applications of semantics, 2519, 1238-1254.

Jarrar, M., \& Meersman, R. (2009). Ontology engineering - The DOGMA approach. In T. Dillon, E. Chang, R. Meersman, \& K. Sycara (Eds.), Advances in web semantics I, lecture notes in computer science (Vol. 4891, pp. 7-34). Berlin/Heidelberg: Springer.

Jiang, X., \& Tan, A. H. (2009). Learning and inferencing in user ontology for personalized semantic web search. Information Sciences: An International Journal, 179(16), 2794-2808.

Johnson, B., \& Shneiderman, B. (1991). Treemaps: A space filling approach to the visualization of hierarchical information structures. In 2nd IEEE visualization conference (pp. 284-291).

Katifori, A., Halatsis, C., Lepouras, G., Vassilakis, C., \& Giannopoulou, E. (2007). Ontology visualization methods - A survey. ACM Computing Surveys, 39(4).

Kozaki, K., Kitamura, Y., \& Mizoguchi, R. (2005). Developing ontology-based applications using HOZO. Proceedings of the fourth IASTED international conference on computational intelligence, 1, 10-16.

Martinho, R., Varajao, J., \& Domingos, D. (2010). Using the semantic web to define a language for modelling controlled flexibility in software processes. IET Software, $4(6), 396-406$

Mizoguchi, R. (2004). Tutorial on ontological engineering: Advanced course of ontological engineering. New Generation Computing, 22(2), 198-220.

Saffer, J. D., Burnett, V. L., Chen, G., \& van der Spek, P. (2004). Visual analytics in the pharmaceutical industry. IEEE Computer Graphics and Applications, 24(5), $10-15$.

Shiva, S. G., \& Shala, L. A. (2008). Using Semantic Wikis to support software reuse. Journal of Software, 3(4), 1-8

Shneiderman, B. (2008). Extreme visualization: Squeezing a billion records into a million pixels. In Proceedings of the 2008 ACM SIGMOD international conference on management of data (pp. 3-12).

Sicilia, J. J., Sicilia, M. A., Sánchez-Alonso, S., García-Barriocanal, E., \& Pontikaki, M. (2009). Knowledge representation issues in ontology-based clinical knowledge management systems. International Journal of Technology Management, 47(1-3), 191-206.

Soto-Acosta, P., Casado-Lumbreras, C., \& Cabezas-Isla, F. (2010). Shaping human capital in software development teams: The case of mentoring enabled by semantics. IET Software, 4(6), 445-452.

Storey, M. A., Musen, M., Silva, J., Best, C., Ernst, N., Fergerson, R., et al. (2001). Jambalaya: interactive visualization to enhance ontology authoring and knowledge acquisition in Protégé. In Workshop on interactive tools for knowledge capture (K-CAP-2001), Victoria, British Columbia, Canada.

Suárez-Figueroa, M., Brockmans, S., Gangemi, A., Gómez-Pérez, A., Lehmann, J., \& Lewen, H. (2007). Neon modeling components. NeOn deliverable d5.1.1, Neon Project. Technical report. NeOn Project.

Sunagawa, E., Kozaki, K., Kitamura, Y., \& Mizoguchi, R. (2005). A framework for organizing role concepts in ontology development tool: Hozo. In Proceedings of the AAAI fall symposium. Technical report FS-05-08, Arlington, Virginia, USA (pp. 136-143).

Sure, Y., Angele, J., \& Staab, S. (2002). Ontoedit: Guiding ontology development by methodology and inferencing. In Proceedings of on the move to meaningful internet systems 2002: CoopIS, DOA, and ODBASE. Lecture notes in computer science (Vol. 2519, pp. 1205-1222).

Suresh, K., Kumar, S., Prakash, N., \& Rizvi, S. (2008). Role of ontology editors: Ontology design. In Proceedings of IPCV'08.

Tacla, C. A., Freddo, A. R., Paraiso, E. C., Ramos, M. P., \& Sato, G. Y. (2011). Supporting small teams in cooperatively building application domain models. Expert Systems with Applications, 38(2), 1160-1170.

Tappolet, J., Kiefer, C., \& Bernstein, A. (2010). Semantic web enabled software analysis. Web Semantics: Science, Services and Agents on the World Wide Web, $8(2-3), 225-240$.

Telea, A., \& Voinea, L. (2009). Case study: Visual analytics in software product assessments. In Proceedings of the 5th IEEE international workshop on visualizing software for understanding and analysis, 2009, VISSOFT 2009 (pp. 65-72). 
Thomas, J. J., \& Cook, K. A. (2005). Illuminating the path: The research and development agenda for visual analytics. Los Alamitos, CA: IEEE Press.

Tominski, C., Abello, J., Van Ham, F., \& Schumann, H. (2006). Fisheye tree views and lenses for graph visualization. In Proceedings of the international conference on information visualization (pp. 17-24).

Tominski, C., Schulze-Wollgast, P., \& Schumann, H. (2008). Visual methods for analyzing human health data. In N. Wickramasinghe \& E. Geisler (Eds.). Encyclopedia of healthcare information systems (vol. 1, pp. 1357-1364). Hershey, PA: IGI-Global.

Valencia-García, R., García-Sánchez, F., Castellanos-Nieves, D., Fernández-Breis, J. T., \& Toval, A. (2010). Exploitation of social semantic technology for software development team configuration. Software, IET, 4(6), 373-385.
Vrba, P., Radakovič, M., Obitko, M., \& Mařík, V. (2011). Semantic technologies: Latest advances in agent-based manufacturing control systems. International Journal of Production Research, 49(5), 1483-1496.

Uschold, M., \& King, M. (1995). Towards a methodology for building ontologies. In Proceedings of workshop on basic ontological issues in knowledge sharing (Vol. 1 pp. 1-15).

Villalón-Terrazas, B., Ramínez, J., Suárez-Figueroa, M. C., \& Gómez-Pérez, A. (2011) A network of ontology networks for building e-employment advanced systems. Expert Systems with Applications, 38(11), 13612-13624.

Ware, C. (2004). Information visualization: Perception for design. San Francisco, CA: Morgan Kaufmann. 